\title{
The Impact of Lymphedema Therapy on Quality of Life: A Case Report
}

\author{
Cleve Carter III*, PT, DPT, MEd, CSCS, CLT, C/NDT, Alexis Andrews, SPT
}

Department of Physical Therapy, College of Health Sciences, 915 South Jackson Street Montgomery, AL 36104, Alabama State University.

Article Details
Article Type: Research Article
Received date: $15^{\text {th }}$ March, 2021
Accepted date: $16^{\text {th }}$ April, 2021
Published date: $20^{\text {th }}$ April, 2021
"Corresponding Author: Cleve Carter III, PT, DPT, MEd, CSCS, CLT, C/NDT, Department of Physical Therapy, College of
Health Sciences, Alabama State University, 915 South Jackson Street Montgomery, AL 36104, United States. E-mail: ccarter@,
alasu.edu
Citation: Carter III, C., \& Andrews, A. (2021). The Impact of Lymphedema Therapy on Quality of Life: A Case Report. $J$
Rehab Pract Res 2(1):117. https://doi.org/10.33790/jrpr1100117
Copyright: O2021, This is an open-access article distributed under the terms of the Creative Commons Attribution License
4.0, which permits unrestricted use, distribution, and reproduction in any medium, provided the original author and source are
credited.

\begin{abstract}
s
Background and Purpose: Lymphedema is a chronic inflammatory lymphostatic disease known as the buildup of lymph fluid in the fatty tissues that can cause swelling and discomfort in the arms, legs, face, neck, trunk, abdomen, and genitals [1]. Failure of the lymphatic system causes lymphedema [1]. Lymphedema may reduce the quality of life and may progress as well as cause other complications such as cellulitis if untreated [1]. Although there is no cure for lymphedema, there are interventions to reduce or manage the swelling and the discomfort [1]. The purpose of this study is to show that a patient with lymphedema can improve their quality of life significantly with adequate lymphedema therapy interventions using Complete Decongestive Therapy (CDT).
\end{abstract}

Keywords: lymphedema, swelling, extremity, complete decongestive therapy

Method and Procedures: A 47-year-old African American female patient presented to outpatient physical therapy with a report of experiencing bilateral lower extremity swelling for about ten years. To determine the effects of the treatments, the lymphedema therapist examined the patient using the following tests and measures and outcome measurement tools: Bilateral Girth Measurements, the Lymphedema Life Impact Scale Version 2 (v2), and the Global Rating of Change (GRoC) Scale. The patient attended physical therapy sessions 2 times per week for 3 weeks not including the date of initial evaluation. Thus, the patient attended five treatment sessions. The patient actively participated in the creation of the current goal: to decrease bilateral lower extremity swelling.

Results: On discharge assessment ( $6^{\text {th }}$ visit $)$, the patient reported a reduction in pain to $(3 / 10)$. The patient scored a $12 / 68$ on the Lymphedema Life Impact Scale (v2) indicating that her quality of life had improved significantly within the past three weeks since receiving lymphedema therapy services. The patient made excellent progress, demonstrated marked improvements with involved extremities lymphatic circulation, and decreased swelling with girth measurements. The patient reported significant improvements with functional activities of daily living such as standing, sitting to standing, and walking. The patient also reported that her quality of life improved significantly.

\section{Background and Purpose}

Lymphedema is a chronic condition known as the buildup of lymph fluid in the fatty tissues that can cause swelling and discomfort in the arms, legs, face, neck, trunk, abdomen, and genitals [1]. Lymphedema can be either primary or secondary [1]. Primary Lymphedema is a rare, inherited condition such as Milroy's disease or Meige's disease that causes problems with the development of lymph vessels in the body [1],. Secondary lymphedema is any condition or procedure that damages your lymph nodes or lymph vessels [1].The most common cause of lymphedema is due to the removal of or damage to the lymph nodes as part of cancer treatment [2]. Trauma to the lymph vessels can also cause lymphedema [1]. Major risk factors of developing secondary lymphedema include older age, excessive weight or obesity and rheumatoid or psoriatic arthritis $[1,2]$. Untreated or incorrectly treated lymphedema reduces the quality of life and may cause the patient many complications [3].

Several studies indicate that patients affected by chronic edema of the lower extremities reported a higher impact on quality of life than individuals with upper extremity lymphedema $[1,4]$. Lymphedema in the lower extremity can impede one's independence and functionality which results in their quality of life diminishing. Studies evaluating functionality and quality of life in lymphedema do so using assessment methods such as questionnaires and physical tests to identify and quantify issues related to the disease and its effects on the different domains of patients' lives [5].

Although lymphedema may diagnosed based on clinical manifestations, a physician may order imaging or test such as a MRI scan, CT scan, Doppler ultrasound or a lymphoscintigraphy $[1,3]$ There is no cure for lymphedema; yet, there are interventions to reduce and manage the swelling and control the pain or discomfort associated with lymphedema. Physical therapists or certified lymphedema therapists may introduce therapeutic exercises that improve the circulation of lymphatic fluid while also preparing the limb for everyday activities [1,3]. Patient education regarding compression garments, therapeutic exercise, self-care, self-manual lymph drainage, and self-compression bandaging is important when teaching patients about the correct fit the garment should have around the limb [1,3].

\section{Methods and Procedures}

A 47-year-old African American female presented to outpatient physical therapy with a report of experiencing bilateral lower extremity swelling for about ten years. The patient stated that the 
onset of swelling occurred after she fractured her left tibia and fibula ten years ago. The patient reported that she had marked discomfort with walking forward due to bilateral lower extremity swelling. The clinician discussed the examination, evaluation, and plan of care process with the patient. The clinician obtained permission to use gathered data to write a case report. To determine the effects of the treatments, the clinician used the following tests and measures and outcome measurement tools: Bilateral Girth Measurements, the Lymphedema Life Impact Scale (v2), and the Global Rating of Change (GRoC) Scale. The clinician was a certified lymphedema therapist with over 10,000 hours evaluating and treating lymphedema.

On the initial examination, the patient reported (6/10) pain level in both lower extremities. The patient reported that she had never had lymphedema therapy prior to her first visit but that she has worn compression stockings without effect. The patient stated that her left lower extremity swelling is always worse than her right lower extremity swelling. Girth measurement of the left lower extremity was a total circumference of $195.2 \mathrm{~cm}$ from the metatarsal heads to the knee and the right lower extremity was total circumference of $191.9 \mathrm{~cm}$ from the metatarsal heads to the knee during initial evaluation. The clinical took circumferential measurements around the metatarsal heads of each foot, the heel of each foot, the ankle (lateral malleolus), mid-calf, and 5 centimeters below knee joint articulation of each lower extremity. The patient scored a 46/68 on the Lymphedema Life Impact Scale (v2) on the initial evaluation. The GRoC was not administered on the initial evaluation; however, it was conducted on the sixth (discharge assessment) session. The patient attended physical therapy sessions for interventions 2 times per week for 3 weeks. Interventions other than patient education were not performed the date of the initial evaluation. The patient actively participated in the creation of the current and primary goal: to decrease bilateral lower extremity swelling.

On the first visit (initial examination), the patient received education on lymphatic system anatomy and involved anatomy with the use of an iPad for visual learning. The patient also received education on indications for Complete Decongestive Therapy (CDT): manual lymph drainage (MLD), compression bandaging, therapeutic exercise, self-care/management, and education. The patient also received instructions for performing lymph drainage exercises as her home exercise program. On the second through fifth visits, the patient received each component of CDT. The patient received MLD using the Vodder Techniques on bilateral lower extremity from feet to knee including the abdominal and cervical sequences and other preparatory sequences to improve lymphatic circulation. The patient also received bilateral lower extremity manual compression bandaging using soft compress liners and multiple short stretch bandages from feet to knees to improve lymphatic circulation. The patient also reported performing lymph drainage exercises for the lower extremity daily. By the third visit, the patient made good progress and demonstrated marked improvements with decreased swelling in involved extremities. The patient had a decrease in swelling of left lower extremity total circumference by $-12.6 \mathrm{~cm}$ and right lower extremity by $-9.4 \mathrm{~cm}$ when compared to measurements on the initial evaluation.

\section{Results}

On discharge assessment ( $6^{\text {th }}$ visit), the patient reported a reduced (3/10) pain level. Per GRoC, the patient reported "A very great deal better" with overall functional performance with functional activities of daily living from the time physical therapy began until now. The patient stated that she could walk forward independently without discomfort and with none to minimal swelling. On the day of discharge, the girth measurement of the left lower extremity total circumference from the foot to the knee was $180.9 \mathrm{~cm}$ and the right lower extremity total circumference from the foot to the knee was $178.9 \mathrm{~cm}$. The patient scored a $12 / 68$ on the Lymphedema Life
Impact Scale (v2) indicating that her quality of life had improved significantly within the past three weeks since receiving lymphedema therapy. The patient made excellent progress and demonstrated marked improvements with involved extremities decreased swelling. The patient achieved $100 \%$ of established goals by the sixth visit. The patient reported that she was $100 \%$ satisfied with the outcomes.

Conflict of Interest: The authors report no conflict of interest.

\section{References}

1. Földi E., Földi M. Földi's Textbook of Lymphology for Physicians and Lymphedema Therapists. 3rd ed. New York, NY: Elsevier; 2012.

2. Vojá-ková, N., Fialová, J., \& Hercogová, J. Management of lymphedema. Dermatologic Therapy, 2012;25(4), 352-357. doi:10.1111/j.1529-8019.2012.01534.x

3. Lymphedema. (2017, December 21). Retrieved March 08, 2021, from https://www.mayoclinic.org/diseases-conditions/ lymphedema/symptoms-causes/syc-20374682

4. Zuther, J. Lymphedema and its impact on quality of life. (2020, April 10). Retrieved March 08, 2021, from https:// www.lymphedemablog.com/2020/04/10/lymphedema-and-itsimpact-on-quality-of-life/

5. Pedrosa, B., Maia, J. N., Ferreira, A., de Araújo, M., Montenegro, E., da Silva, F. L., de Castro, C., \& Andrade, M. Functionality and quality of life of patients with unilateral lymphedema of a lower limb: a cross-sectional study. Jornal vascular brasileiro, 2019; 18, e20180066. https://doi.org/10.1590/1677-5449.006618 